\title{
Products of prime powers in binary recurrence sequences: part I. The hyperbolic case, with an application to the generalized Ramanujan-Nagell equation
}

Citation for published version (APA):

Pethö, A., \& Weger, de, B. M. M. (1986). Products of prime powers in binary recurrence sequences : part I. The hyperbolic case, with an application to the generalized Ramanujan-Nagell equation. Mathematics of Computation, 47(176), 713-727. https://doi.org/10.1090/S0025-5718-1986-0856715-5, https://doi.org/10.2307/2008185

DOI:

10.1090/S0025-5718-1986-0856715-5

$10.2307 / 2008185$

Document status and date:

Published: 01/01/1986

Document Version:

Publisher's PDF, also known as Version of Record (includes final page, issue and volume numbers)

Please check the document version of this publication:

- A submitted manuscript is the version of the article upon submission and before peer-review. There can be important differences between the submitted version and the official published version of record. People interested in the research are advised to contact the author for the final version of the publication, or visit the $\mathrm{DOI}$ to the publisher's website.

- The final author version and the galley proof are versions of the publication after peer review.

- The final published version features the final layout of the paper including the volume, issue and page numbers.

Link to publication

\footnotetext{
General rights

- You may freely distribute the URL identifying the publication in the public portal. follow below link for the End User Agreement:

www.tue.nl/taverne

Take down policy

If you believe that this document breaches copyright please contact us at:

openaccess@tue.nl

providing details and we will investigate your claim.
}

Copyright and moral rights for the publications made accessible in the public portal are retained by the authors and/or other copyright owners and it is a condition of accessing publications that users recognise and abide by the legal requirements associated with these rights.

- Users may download and print one copy of any publication from the public portal for the purpose of private study or research.

- You may not further distribute the material or use it for any profit-making activity or commercial gain

If the publication is distributed under the terms of Article $25 \mathrm{fa}$ of the Dutch Copyright Act, indicated by the "Taverne" license above, please 


\title{
Products of Prime Powers in Binary Recurrence Sequences Part I: The Hyperbolic Case, with an Application to the Generalized Ramanujan-Nagell Equation
}

\author{
By A. Pethö and B. M. M. de Weger
}

\begin{abstract}
We show how the Gelfond-Baker theory and diophantine approximation techniques can be applied to solve explicitly the diophantine equation $G_{n}=w p_{1}^{m_{1}} \cdots p_{t}^{m_{t}}$ (where $\left\{G_{n}\right\}_{n=0}^{\infty}$ is a binary recurrence sequence with positive discriminant), for arbitrary values of the parameters. We apply this to the equation $x^{2}+k=p_{1}^{z_{1}} \cdots p_{t}^{z_{t}}$, which is a generalization of the Ramanujan-Nagell equation $x^{2}+7=2^{z}$. We present algorithms to reduce upper bounds for the solutions of these equations. The algorithms are easy to translate into computer programs. We present an example which shows that in practice the method works well.
\end{abstract}

1. Introduction. The Gelfond-Baker method is one of the most useful tools in the theory of diophantine equations. It has been used to prove effectively computable upper bounds for the solutions of many diophantine problems (cf. Baker [1], Shorey and Tijdeman [17]). However, the derived upper bounds are so large that in many cases it is hopeless to compute all solutions, even with the fastest present-day computers. It seems likely that refinements of the Gelfond-Baker method will not be able to change this situation essentially in the near future.

In those cases where this method has been applied successfully to find all solutions of a certain equation, this has been achieved by reducing the upper bounds considerably, using diophantine approximation techniques (cf. Stroeker and Tijdeman [19]), or by making use of special properties of the diophantine problem (cf. Pethö [12], [13]). These reduced bounds are in practice always small enough to admit enumeration of the remaining possibilities.

In this paper we present such a reduction algorithm for the following problem. Let $A, B, G_{0}, G_{1}$ be integers, and let the recurrence sequence $\left\{G_{n}\right\}_{n=0}^{\infty}$ be defined by

$$
G_{n+1}=A G_{n}-B G_{n-1} \quad(n=1,2, \ldots) \text {. }
$$

Put $\Delta=A^{2}-4 B$, and assume that $\Delta>0$, and that the sequence is not degenerate. Let $w$ be a nonzero integer, and let $p_{1}, \ldots, p_{t}$ be distinct prime numbers. We study the diophantine equation

$$
G_{n}=w p_{1}^{m_{1}} \cdots p_{t}^{m_{t}}
$$

Received October 25, 1985.

1980 Mathematics Subject Classification (1985 Revision). Primary 11B37, 11D61, 11J87, 11Y50. 
in nonnegative integers $n, m_{1}, \ldots, m_{t}$. It was shown by Mahler [9] that (1.1) has only finitely many solutions, and Schinzel [16] has given an effectively computable upper bound for the solutions.

Mignotte [11] indicated how (1.1) with $t=1$ can in some instances be solved by congruence techniques. It is, however, not clear that his method will work for any equation (1.1) with $t=1$. Moreover, his method seems not to be generalizable for $t>1$. Pethö [14] has given a reduction algorithm, based on the Gelfond-Baker method, to treat (1.1) in the case $w=t=1$.

Our reduction algorithm is based on a simple case of $p$-adic diophantine approximation. We shall give explicit upper bounds for the solutions of (1.1) which are small enough to admit the practical application of the reduction algorithm, if the parameters of the equation are not too large.

Pethö [14] pointed out that essentially better upper bounds hold for all but possibly one solution. The reduction algorithm is of course independent of the theoretical upper bounds: it can be used to reduce any upper bound. It also works well for rather small bounds.

The generalized Ramanujan-Nagell equation

$$
x^{2}+k=p_{1}^{z_{1}} \cdots p_{t}^{z_{t}}
$$

( $k$ a fixed integer, $p_{1}, \ldots, p_{t}$ fixed primes) in nonnegative integers $x, z_{1}, \ldots, z_{t}$, can be reduced to a finite number of equations of type (1.1). Equation (1.2) with $t=1$ has a long history (cf. Hasse [5] and Beukers [2]) and interesting applications in coding theory (cf. Bremner et al. [3], MacWilliams and Sloane [8], Tzanakis and Wolfskill [21], [22]). Examples of Eq. (1.2) have been solved by the Gelfond-Baker method by D. C. Hunt and A. J. van der Poorten in an unpublished paper. They used complex, not $p$-adic linear forms in logarithms. As far as we know, none of the proposed methods to treat (1.2) gives rise to an algorithm which works for arbitrary values of $k$ and $p_{i}$, whereas Tzanakis' elementary method [20] seems to be the only one that can be generalized to $t>1$. Our method has both properties. Evertse [4] has proved that the number of solutions of (1.2) does not exceed $3 \times 7^{4 t+6}$.

In Section 2 we give the necessary background on $p$-adic numbers and logarithms, and on linear binary recurrence sequences. In Section 3 we apply a theorem of Schinzel to find upper bounds for the solutions of (1.1). This result holds for positive $\Delta$ as well as for negative, whereas in the remaining sections we restrict ourselves to positive $\Delta$. Section 4 includes the reduction algorithm and gives a worked-out example. In Section 5 we study (1.2). As an example we determine all integers $x$ such that $x^{2}+7$ has no prime factors larger than 20. Finally, we note that our method can be applied to a somewhat more general type of quadratic/exponential diophantine equation.

In the second part of this paper ([23]), the second-named author intends to study Eq. (1.1) for negative discriminant.

\section{Preliminaries.}

2A. p-Adic Numbers and Logarithms. For the convenience of the reader we quote the facts that we use from the theory of $p$-adic numbers and functions. An extensive treatment of this theory is given in Koblitz [6]. 
Let $p$ be a prime number, and $\Delta$ a nonsquare integer (positive or negative). We study the extension $\mathbb{Q}_{p}(\sqrt{\Delta})$ of the field of $p$-adic numbers $\mathbb{Q}_{p}$. Either $\sqrt{\Delta} \in \mathbb{Q}_{p}$, or $\mathbb{Q}_{p}(\sqrt{\Delta})=\mathbb{Q}_{p}+\sqrt{\Delta} \mathbb{Q}_{p}$. In the former case, $\mathbb{Q}_{p}(\sqrt{\Delta})=\mathbb{Q}_{p}$, and the $p$-adic order $\operatorname{ord}_{p}(\xi)$ is well defined for any $\xi \in \mathbb{Q}_{p}(\sqrt{\Delta})$, as usual. In the latter case, it is defined as follows. Let $\xi$ and $\xi^{\prime}$ be the roots of $x^{2}+a x+b, a, b \in \mathbb{Q}_{p}$. Then we define

$$
\operatorname{ord}_{p}(\xi)=\operatorname{ord}_{p}\left(\xi^{\prime}\right)=\frac{1}{2} \operatorname{ord}_{p}(b) \text {. }
$$

Notice that this formula is not necessarily true if $\sqrt{\Delta} \in \mathbb{Q}_{p}$.

On $\mathbb{Q}_{p}(\sqrt{\Delta})$ there is a logarithmic function, denoted by $\log _{p}$. It is defined for all $\chi \in \mathbb{Q}_{p}(\sqrt{\Delta})$ with ord $p(\chi)=0$, as follows. Let $k$ be the smallest positive integer such that $\xi=\chi^{k}-1$ has $\operatorname{ord}_{p}(\xi) \geqslant \frac{1}{2}$. Then

$$
\log _{p}(\chi)=\frac{1}{k} \log _{p}(1+\xi)=\frac{1}{k}\left(\xi-\xi^{2} / 2+\xi^{3} / 3-\xi^{4} / 4+\cdots\right) .
$$

Notice that this series converges, and is useful for computing $\log _{p}(\xi)$ to any desired degree of accuracy. Note that $\log _{p}(\chi) \in \mathbb{Q}_{p}(\sqrt{\Delta}), \log _{p}\left(\chi_{1} \chi_{2}\right)=\log _{p}\left(\chi_{1}\right)+$ $\log _{p}\left(\chi_{2}\right)$, and $\log _{p}\left(\chi^{k}\right)=k \log _{p}(\chi)$ hold for all $k \in \mathbb{Z}, \quad \chi, \chi_{1}, \chi_{2} \in \mathbb{Q}_{p}(\sqrt{\Delta})$. Further, $\log _{p}(\chi)=0$ if and only if $\chi$ is a root of unity.

Let $\chi=1+\xi \in \mathbb{Q}_{p}(\sqrt{\Delta})$ not be a root of unity, such that

$$
\operatorname{ord}_{p}(\xi) \geqslant \begin{cases}3 / 2 & \text { if } p=2 \\ 1 & \text { if } p=3 \\ 1 / 2 & \text { if } p \geqslant 5\end{cases}
$$

Then

$$
\operatorname{ord}_{p}\left(\log _{p}(\chi)\right)=\operatorname{ord}_{p}(\xi) \text {. }
$$

Suppose that $\sqrt{\Delta} \notin \mathbb{Q}_{p}$. Let $\alpha=a+b \sqrt{\Delta}, a, b \in \mathbb{Q}_{p}$, and let $\beta=a-b \sqrt{\Delta}$ be its conjugate. Then notice that $\log _{p}(\alpha)$ and $\log _{p}(\beta)$ are conjugates. Hence,

$$
\log _{p}(\alpha / \beta) \in \sqrt{\Delta} \mathbb{Q}_{p}
$$

Let $k$ be the smallest positive integer such that

$$
\left(\frac{\alpha}{\beta}\right)^{k}= \pm \frac{1+\xi}{1-\xi}
$$

with $\operatorname{ord}_{p}(\xi) \geqslant \frac{1}{2}$. Then we can compute $\log _{p}(\alpha / \beta)$ by the series

$$
\log _{p}(\alpha / \beta)=\frac{2}{k}\left(\xi+\xi^{3} / 3+\xi^{5} / 5+\cdots\right) .
$$

2B. Binary Recurrences. Let $A, B, G_{0}, G_{1}$ be given integers. Let the sequence $\left\{G_{n}\right\}_{n=0}^{\infty}$ be defined by

$$
G_{n+1}=A G_{n}-B G_{n-1} \quad(n=1,2, \ldots) .
$$

Let $\alpha, \beta$ be the roots of $x^{2}-A x+B$. We assume that $\Delta=A^{2}-4 B$ is not a square, and that $\alpha / \beta$ is not a root of unity (i.e., the sequence is not degenerate). Put

$$
\lambda=\frac{G_{1}-G_{0} \beta}{\alpha-\beta}, \quad \mu=\frac{G_{0} \alpha-G_{1}}{\alpha-\beta} .
$$

Then $\lambda$ and $\mu$ are conjugates. It is well known that for all $n \geqslant 0$

$$
G_{n}=\lambda \alpha^{n}+\mu \beta^{n} \text {. }
$$


Since our aim is to solve Eq. (1.1), we see from (2.5) that we may assume without loss of generality that $\left(G_{0}, G_{1}\right)=\left(G_{1}, B\right)=(A, B)=1$.

LEMMA 2.1. Let $n, m_{1}, \ldots, m_{t}$ be a solution of (1.1). Then, with the above assumptions, we have for $i=1, \ldots, t$ : either $m_{i}=0$, or $n=0$, or

$$
\operatorname{ord}_{p_{t}}(\alpha)=\operatorname{ord}_{p_{t}}(\beta)=0, \quad \operatorname{ord}_{p_{t}}(\lambda)=\operatorname{ord}_{p_{t}}(\mu)=-\frac{1}{2} \operatorname{ord}_{p_{t}}(\Delta) \leqslant 0 .
$$

Proof. Suppose $p_{i} \mid B$. Then $p_{i}+A$, hence, from (2.5) and $\left(B, G_{1}\right)=1, p_{i}+G_{n}$ for all $n>0$. Thus, $m_{l}=0$ or $n=0$. Next suppose $p_{i}+B$. Then, by $\alpha \beta=B$,

$$
\operatorname{ord}_{p_{i}}(\alpha)+\operatorname{ord}_{p_{i}}(\beta)=\operatorname{ord}_{p_{i}}(B)=0 \text {. }
$$

Now, $\alpha$ and $\beta$ are algebraic integers, so $\operatorname{ord}_{p_{t}}(\alpha)$ and $\operatorname{ord}_{p_{t}}(\beta)$ are nonnegative. It follows that they are zero.

Put $E=-\lambda \mu \Delta$. Note that $E \in \mathbb{Z}$, and for all $n \geqslant 0$

$$
G_{n+1}^{2}-A G_{n} G_{n+1}+B G_{n}^{2}=E B^{n} \text {. }
$$

Suppose that $p_{l} \mid E$; then we infer that $p_{i}+G_{n}$ for all $n$, since $\left(G_{0}, G_{1}\right)=1$. Hence $m_{i}=0$. Next, suppose $p_{i}+E$; then

$$
\operatorname{ord}_{p_{i}}(\lambda \sqrt{\Delta})+\operatorname{ord}_{p_{i}}(\mu \sqrt{\Delta})=\operatorname{ord}_{p_{i}}(E)=0 .
$$

Since $\lambda \sqrt{\Delta}$ and $\mu \sqrt{\Delta}$ are algebraic integers (cf. (2.6)), the result follows.

From Lemma 2.1 it follows that we may assume without loss of generality that (2.8) holds for $i=1, \ldots, t$. Of course, we may also assume that ord $_{p_{t}}(w)=0$ for $i=1, \ldots, t$. The special case $t=0$ in Eq. (1.1) is trivial if $\Delta>0$, and demands special treatment if $\Delta<0$.

Finally, we prove a simple auxiliary lemma.

LEMMA 2.2. Let $a \geqslant 0, h \geqslant 1, b>\left(e^{2} / h\right)^{h}$, and let $x \in \mathbb{R}$ be the largest solution of $x=a+b(\log x)^{h}$. Then,

$$
x<2^{h}\left(a^{1 / h}+b^{1 / h} \log \left(h^{h} b\right)\right)^{h} .
$$

Proof. By $\left(z_{1}+z_{2}\right)^{1 / h} \leqslant z_{1}^{1 / h}+z_{2}^{1 / h}$ we infer

$$
x^{1 / h} \leqslant a^{1 / h}+c \log \left(x^{1 / h}\right),
$$

where $c=h b^{1 / h}>e^{2}$. Put $x^{1 / h}=(1+y) c \log c$; then $y>0$. Now,

$$
\begin{aligned}
(1+y) c \log c & =x^{1 / h} \leqslant a^{1 / h}+c \log (1+y)+c \log c+c \log \log c \\
& <a^{1 / h}+c y+c \log c+c \log \log c,
\end{aligned}
$$

hence,

It follows, by $c>e^{2}$, that

$$
y c(\log c-1)<a^{1 / h}+c \log \log c .
$$

$$
\begin{aligned}
x^{1 / h} & =c \log c+y c \log c<c \log c+\frac{\log c}{\log c-1}\left(a^{1 / h}+c \log \log c\right) \\
& <2\left(a^{1 / h}+c \log c\right) .
\end{aligned}
$$

3. Application of Schinzel's Theorem. In this section, $\Delta$ may be positive or negative. We quote a result of Schinzel [16] and apply it to (1.1).

Let $p$ be prime. Let $D$ be the discriminant of $\mathbb{Q}(\sqrt{\Delta}), \xi$ and $\chi$ nonzero elements of $\mathbb{Q}(\sqrt{\Delta})$. Put $\xi=\xi^{\prime \prime} / \xi^{\prime}, \chi=\chi^{\prime \prime} / \chi^{\prime}$, where $\xi^{\prime}, \xi^{\prime \prime}, \chi^{\prime}, \chi^{\prime \prime}$ are algebraic integers. Put

$$
L=\log \max \left\{|e D|^{1 / 4},\left\|\xi^{\prime} \chi^{\prime}\right\|,\left\|\xi^{\prime} \chi^{\prime \prime}\right\|,\left\|\xi^{\prime \prime} \chi^{\prime}\right\|,\left\|\xi^{\prime \prime} \chi^{\prime \prime}\right\|\right\}
$$


where $\|\gamma\|$ is the maximal absolute value of the conjugates of $\gamma$. Let $\mathfrak{p}$ be a prime ideal of $\mathbb{Q}(\sqrt{\Delta})$ with norm $p^{\rho}$. Put

$$
\psi=\frac{2}{\rho \log p}, \quad \phi=\operatorname{ord}_{\mathfrak{p}}(p) .
$$

THEOREM 3.1 (SCHINZEL). If $\xi$ or $\chi$ is a $\mathfrak{p}$-adic unit and $\xi^{n} \neq \chi^{m}$, then

$$
\operatorname{ord}_{\mathfrak{p}}\left(\xi^{n}-\chi^{m}\right)<10^{6} \psi^{7} \phi^{-2} L^{4} p^{4 \rho+4}\left(\log \max (|m|,|n|)+\phi L p^{\rho}+2 / L\right)^{3} \text {. }
$$

We apply this to Eq. (1.1) as follows. Let $n_{0} \geqslant 2$, and put

$$
L=\log \max \left\{|e D|^{1 / 4},|\alpha \lambda \sqrt{\Delta}|,|\alpha \mu \sqrt{\Delta}|,|\beta \lambda \sqrt{\Delta}|,|\beta \mu \sqrt{\Delta}|\right\} .
$$

Let $d$ be the squarefree part of $\Delta$. For $i=1, \ldots, t$, put

$$
\begin{aligned}
& \phi_{i}=2 \text { if } p_{i} \mid d, \phi_{i}=1 \text { otherwise, } \\
& \rho_{i}=2 \text { if } p_{i}=2, d \equiv 5(\bmod 8) \text { or if } p_{i}>2,\left(\frac{d}{p_{i}}\right)=-1 \\
& \rho_{i}=1 \text { otherwise, } \\
& C_{1, l}=10^{6}\left(\frac{2}{\rho_{i} \log p_{i}}\right)^{7} \phi_{i}^{-3} L^{4} p_{t}^{4 \rho_{\imath}+4}\left(1+\frac{\phi_{i} L p_{l}^{\rho_{l}}+2 / L}{\log n_{0}}\right)^{3} .
\end{aligned}
$$

LEMMA 3.2. The solutions of (1.1) with $n \geqslant n_{0}$ satisfy

$$
m_{i}<C_{1, i}(\log n)^{3} \quad(i=1, \ldots, t) .
$$

Proof. Rewrite (1.1), using (2.7), as

$$
\left(\frac{\alpha}{\beta}\right)^{n}-\left(\frac{-\mu}{\lambda}\right)=\frac{w}{\lambda} \beta^{-n} p_{1}^{m_{1}} \cdots p_{t}^{m_{t}}
$$

Then, by (2.8),

$$
m_{i} \leqslant m_{i}-\operatorname{ord}_{p_{i}}(\lambda)=\operatorname{ord}_{p_{i}}\left(\frac{w}{\lambda} \beta^{-n} p_{1}^{m_{1}} \cdots p_{t}^{m_{t}}\right)=\operatorname{ord}_{p_{\imath}}\left(\left(\frac{\alpha}{\beta}\right)^{n}-\left(\frac{-\mu}{\lambda}\right)\right) .
$$

Put $\xi^{\prime \prime}=\alpha, \xi^{\prime}=\beta, \chi^{\prime \prime}=\mu \sqrt{\Delta}, \chi^{\prime}=-\lambda \sqrt{\Delta}$. Then, from Theorem 3.1 we find, using $\operatorname{ord}_{p_{i}}(x)=\phi_{i} \operatorname{ord}_{p_{i}}(x)$,

$$
m_{l}<10^{6}\left(\frac{2}{\rho_{i} \log p_{i}}\right)^{7} \phi_{i}^{-3} L^{4} p_{i}^{4 \rho_{i}+4}\left(\log n+\phi_{l} L p_{i}^{\rho_{l}}+2 / L\right)^{3},
$$

from which the result follows, since $n \geqslant n_{0}$.

Remark. Instead of Schinzel's result, we could have used Theorem 1 of van der Poorten [15]. Then we would have found $m_{1}<C_{1, i}^{\prime} \log n$. This is better in $\log n$, and $C_{1, i}^{\prime}$ has better asymptotic dependence on $p_{\imath}$ than $C_{1, i}$. But for $p_{\imath}$ up to, say, $10^{4}$, Schinzel's result is sharper.

\section{How to Solve (1.1).}

4A. Bounds for the Solutions. In this subsection, let $\Delta>0$. Note that $|\alpha| \neq|\beta|$, since $\left\{G_{n}\right\}_{n=0}^{\infty}$ is not degenerate. So we may assume $|\alpha|>|\beta|$. Let $C_{1}=$ $\max \left(C_{1,1}, \ldots, C_{1, t}\right), m=\max \left(m_{1}, \ldots, m_{t}\right)$, and $P=p_{1} \cdots p_{t}$.

The following theorem gives explicit upper bounds for the solutions of (1.1). We do not claim that this result is original or best possible. It just gives correct and rather small upper bounds. 
THEOREM 4.1. Let $\Delta>0$ and $|\alpha|>|\beta|$, and let the assumptions of Subsection 2B hold. Let $n_{0}>\max (2, \log |\mu / \lambda| / \log |\alpha / \beta|)$. Put

$$
\begin{aligned}
& \gamma=|\lambda / w|-|\mu / w||\alpha / \beta|^{-n_{0}}, \\
& C_{2}=\log P /\left(\log |\alpha|+\frac{1}{n_{0}} \min (0, \log \gamma)\right), \\
& C_{3}=\max \left\{8 C_{1}\left(\log 27 C_{1} C_{2}\right)^{3}, 841 C_{1}\right\} .
\end{aligned}
$$

Then all solutions $n, m_{1}, \ldots, m_{t}$ of (1.1) with $n \geqslant n_{0}$ satisfy

$$
n<\sum_{i=1}^{t} m_{i} \frac{\log p_{i}}{\log |\alpha|}-\frac{\log \gamma}{\log |\alpha|} .
$$

Moreover, $n<C_{2} C_{3}$ and $m<C_{3}$.

Proof. Rewrite (1.1), using (2.7), as

$$
\frac{\lambda}{w}+\frac{\mu}{w}\left(\frac{\alpha}{\beta}\right)^{-n}=p_{1}^{m_{1}} \cdots p_{t}^{m_{t}} \alpha^{-n}
$$

Note that $\gamma>0$, by the choice of $n_{0}$. It follows that

$$
p_{1}^{m_{1}} \cdots p_{t}^{m_{t}}|\alpha|^{-n} \geqslant \gamma
$$

and we infer (4.1) immediately. From (4.1) we obtain $n<C_{2} m$. By Lemma 3.2 we now have

$$
m<C_{1}(\log n)^{3}<C_{1}\left(\log C_{2} m\right)^{3} .
$$

If $C_{1} C_{2}>\left(e^{2} / 3\right)^{3}$, we apply Lemma 2.2 with $a=0, b=C_{1} C_{2}, h=3$; then we find $m<8 C_{1}\left(\log 27 C_{1} C_{2}\right)^{3}$. If $C_{1} C_{2} \leqslant\left(e^{2} / 3\right)^{3}$, then

$$
n<C_{2} m<C_{1} C_{2}(\log n)^{3} \leqslant\left(e^{2} / 3\right)^{3}(\log n)^{3},
$$

from which we deduce $n<12564$. Now, $m<C_{1}(\log n)^{3}<841 C_{1}$.

4B. Notations and Preliminary Lemmas. We introduce some notations. Until further notice, $\Delta$ may be positive or negative. Let for $i=1, \ldots, t$,

$$
\begin{gathered}
e_{i}=-\operatorname{ord}_{p_{i}}(\lambda), \quad f_{i}=\operatorname{ord}_{p_{\imath}}\left(\log _{p_{i}}(\alpha / \beta)\right), \quad g_{i}=f_{i}-e_{\imath}, \\
\theta_{i}=-\log _{p_{i}}(-\lambda / \mu) / \log _{p_{i}}(\alpha / \beta) .
\end{gathered}
$$

By (2.8), the $p_{i}$-adic logarithms of $\alpha / \beta$ and $\lambda / \mu$ exist. Notice that $\log _{p_{1}}(\alpha / \beta) \neq 0$, since the sequence $\left\{G_{n}\right\}$ is not degenerate. By (2.3), numerator and denominator of $\boldsymbol{\theta}_{\imath}$ are both in $\sqrt{\Delta} \mathbb{Q}_{p_{i}}$, so $\boldsymbol{\theta}_{i} \in \mathbb{Q}_{p_{i}}$. Hence, if $\boldsymbol{\theta}_{i} \neq 0$, we can write

$$
\theta_{i}=\sum_{l=k_{l}}^{\infty} u_{i, l} p_{i}^{l}
$$

where $k_{i}=\operatorname{ord}_{p_{l}}\left(\theta_{i}\right)$, and $u_{i, l} \in\left\{0,1, \ldots, p_{i}-1\right\}$.

The following, almost trivial lemma is at the heart of our reduction algorithm. It localizes the elements of $\left\{G_{n}\right\}$ with many factors $p_{i}$ in terms of the $p_{i}$-adic expansion of $\boldsymbol{\theta}_{i}$. 
LEMMA 4.2. Let $n \in \mathbb{Z}, n \geqslant 0$. If

$$
\operatorname{ord}_{p_{i}}\left(G_{n}\right)+e_{i} \geqslant \begin{cases}3 / 2 & \text { if } p_{i}=2, \\ 1 & \text { if } p_{i}=3, \\ 1 / 2 & \text { if } p_{i} \geqslant 5,\end{cases}
$$

then $\operatorname{ord}_{p_{i}}\left(G_{n}\right)=g_{i}+\operatorname{ord}_{p_{i}}\left(n-\theta_{i}\right)$.

Proof. By (2.8) we have

$$
\operatorname{ord}_{p_{i}}\left(G_{n}\right)+e_{i}=\operatorname{ord}_{p_{i}}\left(\left(\frac{\alpha}{\beta}\right)^{n}-\left(\frac{-\mu}{\lambda}\right)\right)=\operatorname{ord}_{p_{i}}\left(\left(\frac{-\lambda}{\mu}\right)\left(\frac{\alpha}{\beta}\right)^{n}-1\right) .
$$

With $\xi=(-\lambda / \mu)(\alpha / \beta)^{n}-1$, condition (2.1) holds. Hence, from (2.2),

$$
\begin{aligned}
\operatorname{ord}_{p_{i}}\left(G_{n}\right)+e_{i} & =\operatorname{ord}_{p_{i}}\left(n \log _{p_{i}}\left(\frac{\alpha}{\beta}\right)+\log _{p_{i}}\left(\frac{-\lambda}{\mu}\right)\right) \\
& =\operatorname{ord}_{p_{i}}\left(n-\theta_{i}\right)+f_{i} .
\end{aligned}
$$

Before we give the algorithm, we have to exclude some trivial cases.

LEMMA 4.3. If $\operatorname{ord}_{p_{i}}\left(\theta_{i}\right)<0$, then for the solutions of (1.1),

$$
m_{i} \leqslant \max \left(1, \operatorname{ord}_{p_{i}}\left(\log _{p_{i}}(-\lambda / \mu)\right)\right)-e_{i} .
$$

Proof. If $m_{i} \geqslant 3 / 2-e_{i}$, we apply Lemma 4.2, and obtain

$$
m_{i}=f_{i}-e_{i}+\operatorname{ord}_{p_{i}}\left(n-\theta_{i}\right) .
$$

Since $n \in \mathbb{Z}$ and $\operatorname{ord}_{p_{i}}\left(\theta_{i}\right)<0$, we have ord $p_{i}\left(n-\theta_{i}\right)=\operatorname{ord}_{p_{i}}\left(\theta_{i}\right)$. Hence

$$
m_{i}=f_{i}+\operatorname{ord}_{p_{i}}\left(\theta_{i}\right)-e_{i}=\operatorname{ord}_{p_{i}}\left(\log _{p_{i}}(-\lambda / \mu)\right)-e_{i} \text {. }
$$

Thus we may assume that $\operatorname{ord}_{p_{i}}\left(\theta_{i}\right) \geqslant 0$ for $i=1, \ldots, t$. The reduction algorithm is based on the assumption that infinitely many $p_{i}$-adic digits $u_{i, l}$ of $\theta_{i}$ are nonzero. We now show that if this is not the case, then Eq. (1.1) can be solved in an elementary way. From now on, $\Delta>0$.

LEMMA 4.4. There are only finitely many $p_{i}$-adic digits $u_{i, l}$ of $\theta_{i}$ nonzero if and only if there exists a nonnegative integer $r$ such that $G_{n}= \pm R_{n-r}$ or $G_{n}= \pm \kappa S_{n-r}$, where $\kappa=1$ or $\frac{1}{2}$ and

$$
R_{n}=\frac{\alpha^{n}-\beta^{n}}{\alpha-\beta}, \quad S_{n}=\alpha^{n}+\beta^{n}
$$

Proof. By ord $p_{i}\left(\theta_{i}\right) \geqslant 0$ we have $\theta_{i}=r$ for some rational integer $r \geqslant 0$. From the definition of $\boldsymbol{\theta}_{i}$,

$$
\log _{p_{i}}\left(\frac{\alpha}{\beta}\right)^{r}\left(\frac{-\lambda}{\mu}\right)=0,
$$

hence $(\alpha / \beta)^{r}(-\lambda / \mu)$ is a root of unity. Since $\Delta>0$, it is \pm 1 . Hence, $\lambda \alpha^{r}= \pm \mu \beta^{r}$, and we infer

$$
G_{n}=\lambda \alpha^{r}\left(\alpha^{n-r} \pm \beta^{n-r}\right)
$$

Suppose that $\alpha \beta= \pm 1$. Then

$$
\begin{aligned}
& G_{0}=\lambda \alpha^{r}\left(\alpha^{-r} \pm \beta^{-r}\right)= \pm \lambda \alpha^{r}\left(\alpha^{r} \pm \beta^{r}\right) \\
& G_{1}=\lambda \alpha^{r}\left(\alpha^{1-r} \pm \beta^{1-r}\right)= \pm \lambda \alpha^{r}\left(\alpha^{r-1} \pm \beta^{r-1}\right)
\end{aligned}
$$


Notice that

$$
\left(\alpha^{r-1}+\beta^{r-1}, \alpha^{r}+\beta^{r}\right)=(2, \alpha+\beta)=1 \text { or } 2,
$$

and

$$
\left(\alpha^{r-1}-\beta^{r-1}, \alpha^{r}-\beta^{r}\right)=\alpha-\beta .
$$

By $\left(G_{0}, G_{1}\right)=1$ it follows that $\pm \lambda \alpha^{r}=1, \frac{1}{2}$ or $1 /(\alpha-\beta)$, respectively, and the assertion follows.

Suppose next that $|\alpha \beta|>1$. Notice that

$$
G_{0} B\left(\alpha^{r-1} \pm \beta^{r-1}\right)=G_{1}\left(\alpha^{r} \pm \beta^{r}\right)
$$

where all factors are integers. Since $\left(B, G_{1}\right)=1$, we have $\alpha \beta \mid \alpha^{r} \pm \beta^{r}$. Suppose $r>0$. Then it follows that $(\alpha, \beta) \neq 1$, which contradicts $(A, B)=1$. Hence $r=0$, and the assertion follows from (4.2) and $\left(G_{0}, G_{1}\right)=1$.

Thus, in the situation of Lemma 4.4 we have to solve (1.1) for $R_{n}$ and $S_{n}$. These recurrences enjoy the following divisibility properties,

$$
\begin{aligned}
& R_{n} \mid R_{m} \quad \text { if and only if } n \mid m, \\
& S_{n} \mid S_{k n} \text { for odd } k, \\
& \operatorname{ord}_{2}\left(S_{n}\right) \leqslant \operatorname{ord}_{2}\left(S_{3}\right) \text { for all } n \geqslant 1 .
\end{aligned}
$$

Ideas similar to those of Størmer [18], Mahler [10], and Lehmer [7] can be employed to solve (1.1) using these properties. We do not work this out, but we confine ourselves to an illustrative example at the end of this section. It is also possible to derive elementary upper bounds in this case. Note that $\theta_{i}=r$ holds for all $i$ with the same $r$. Thus, by Lemma 4.2,

$$
m_{\imath} \leqslant \max \left(g_{i}+\operatorname{ord}_{p_{l}}(n-r), 1-e_{\imath}\right) \leqslant g_{\imath}+1+\operatorname{ord}_{p_{l}}(n-r) .
$$

Then we have, by (4.1),

$$
n \log |\alpha|<\sum_{i=1}^{t}\left(g_{i}+1\right) \log p_{i}-\log \gamma+\log |n-r|,
$$

from which a good upper bound for $n$ can be derived.

So we assume in the sequel that infinitely many $p_{i}$-adic digits of $\boldsymbol{\theta}_{i}$ are nonzero.

4C. The Reduction Algorithm. Let all the above assumptions hold. Let $N$ be a positive real number such that we are only interested in the solutions $n, m_{1}, \ldots, m_{t}$ of (1.1) with $n<N$. For example, take $N=C_{2} C_{3}$, as in Theorem 4.1.

Algorithm A (reduces given upper bounds for the solutions of (1.1)).

Input: $\alpha, \beta, \lambda, \mu, w, p_{1}, \ldots, p_{t}, N$

Output: new, better upper bounds $M_{l}$ and $N^{*}$ for $m_{l}(i=1, \ldots, t)$ and $n$.

(i) (initialization) Choose an $n_{0} \geqslant 0$ such that $n_{0}>\log |\mu / \lambda| / \log |\alpha / \beta|$;

$$
\begin{aligned}
& \gamma:=|\lambda / w|-|\mu / w||\alpha / \beta|^{-n_{0}} ; \\
& g_{t}:=\operatorname{ord}_{p_{t}}(\lambda)+\operatorname{ord}_{p_{t}}\left(\log _{p_{t}}(\alpha / \beta)\right) \\
& h_{t}:=\operatorname{ord}_{p_{t}}(\lambda)+\left\{\begin{array}{cc}
3 / 2 & \text { if } p_{t}=2 \\
1 & \text { if } p_{i}=3 \\
1 / 2 & \text { if } p_{i} \geqslant 5
\end{array}\right\} \quad(i=1, \ldots, t) \\
& g:=\gamma / \prod_{t=1}^{t} p_{i}^{g_{i}} ; N_{0}:=N
\end{aligned}
$$


(ii) (computation of the $\theta_{l}$ 's) Compute for $i=1, \ldots, t$, using (2.4), the first $r_{t}$ $p_{i}$-adic digits $u_{i, l}$ of

$$
\theta_{i}=-\log _{p_{l}}(-\lambda / \mu) / \log _{p_{l}}(\alpha / \beta)=\sum_{l=0}^{\infty} u_{t, l} p_{i}^{\prime},
$$

where $r_{i}$ is so large that $p_{t}^{r_{t}} \geqslant N_{0}$ and $u_{t, r_{t}} \neq 0$;

(iii) (further initialization, start outer loop) $s_{t, 0}:=r_{i}+1(i=1, \ldots, t) ; j:=1$;

(iv) (start inner loop) $i:=1 ; K_{J}:=$.false.;

(v) (computation of the new bounds for $m_{i}$ )

$s_{i, j}:=\min \left\{s \in \mathbb{Z}: s \geqslant 0\right.$ and $p_{i}^{s} \geqslant N_{j-1}$ and $\left.u_{l, s} \neq 0\right\}$;

if $s_{l, j}<s_{l, j-1}$ then $K_{j}:=$.true.;

(vi) (terminate inner loop) if $i<t$ then $i:=i+1$, goto (v);

(vii) (computation of the new bound for $n$ )

$N_{j}:=\min \left\{N_{J-1},\left(\sum_{i=1}^{t} s_{i, j} \log p_{i}-\log g\right) / \log |\alpha|\right\} ;$

(viii) (terminate outer loop)

$$
\begin{aligned}
& \text { if } N_{J} \geqslant n_{0} \text { and } K \text {, then } j:=j+1 \text {, goto (iv); } \\
& \text { else } N^{*}:=\max \left({\overline{N_{J}, n_{0}}}\right) \\
& M_{\imath}:=\max \left(h_{\imath}, g_{\imath}+s_{i, J}\right)(i=1, \ldots, t) ; \\
& \text { stop. }
\end{aligned}
$$

THEOREM 4.5. With the above assumptions, Algorithm A terminates. Equation (1.1) has no solutions with $N^{*} \leqslant n<N, m_{l}>M_{t}(i=1, \ldots, t)$.

Proof. Since the $p_{t}$-adic expansion of $\theta_{i}$ is assumed to be infinite, there exist $r_{t}$ with the required properties. It is clear that $s_{t, 1} \leqslant r_{i}<s_{i, 0}$, and that $N_{J} \leqslant N_{J-1}$. So $s_{i, j} \leqslant s_{i, j-1}$ holds for all $j \geqslant 1$. Since $s_{i, j} \geqslant 0$, there is a $j$ such that $N_{j} \leqslant n_{0}$ or $s_{t, j}=s_{t, j-1}$ for all $i=1, \ldots, t$. In the latter case, $K$, remains .false.; in both cases the algorithm terminates.

We prove by induction on $j$ that $m_{\imath} \leqslant g_{\imath}+s_{t, j}(i=1, \ldots, t)$ and $n<N_{\text {, holds }}$ for all $j$. For $j=0$, it is clear that $n<N_{0}$. Suppose $n<N_{j-1}$ for some $j \geqslant 1$. Suppose there exists an $i$ such that $m_{l}>g_{i}+s_{i, j}$. From Lemma 4.2 we have

$$
\operatorname{ord}_{p_{l}}\left(n-\theta_{l}\right)=m_{l}-g_{\imath} \geqslant s_{\imath, J}+1 \text {, }
$$

hence, by $u_{i, s_{i, j}} \neq 0$,

$$
n \geqslant u_{i, 0}+u_{i, 1} p+\cdots+u_{i, s_{t, j}} p^{s_{t, \jmath}} \geqslant p^{s_{t, \jmath}} \geqslant N_{J-1},
$$

which contradicts our assumption. Thus, $m_{l} \leqslant g_{l}+s_{l, J}(i=1, \ldots, t)$. Then from (4.1), it follows that

$$
n<\left(\sum_{i=1}^{t}\left(g_{\imath}+s_{i, j}\right) \log p_{\imath}-\log \gamma\right) / \log |\alpha|,
$$

hence, $n<N_{\text {s }}$.

Remark 1. In general, one expects that $p_{i}^{s_{t, j}}$ will not be much larger than $N_{,}$, i.e., not too many consecutive $p_{i}$-adic digits of $\theta_{t}$ will be zero. Then $N_{J}$ is about as large as $\log N_{J-1}$. In practice, the algorithm will often terminate in three or four steps, near 
to the largest solution. The computation time is polynomial in $t$, the bottleneck of the algorithm is the computation of the $p_{i}$-adic logarithms.

Remark 2. Pethö [14] gives for $t=1$ a different reduction algorithm. For a prime $p_{\imath}$ he computes the function $g(u)$, defined for $u \in \mathbb{N}$ as the smallest index $n \geqslant 0$ such that $G_{n} \neq 0$ and $p_{l}^{u} \mid G_{n}$. Notice that if the $p_{i}$-adic $\operatorname{limit}_{u \rightarrow \infty} g(u)$ exists, then by Lemma 4.2 it is equal to $\theta_{i}$.

Remark 3. If $B= \pm 1$, we can extend the sequence $\left\{G_{n}\right\}_{n=0}^{\infty}$ to negative indices $n$ as follows. For $n<0$, we define

$$
G_{n}=\lambda \alpha^{n}+\mu \beta^{n}=B^{n}\left(\mu \alpha^{|n|}+\lambda \beta^{|n|}\right) \in \mathbb{Z} .
$$

We can solve Eq. (1.1) with $n \in \mathbb{Z}$ not necessarily nonnegative, by applying Algorithm A twice: once for $\left\{G_{n}\right\}_{n=0}^{\infty}$, and once for the sequence $\left\{G_{n}^{\prime}\right\}_{n=0}^{\infty}$, defined by $G_{n}^{\prime}=G_{-n}$. Notice that

$$
\theta_{\imath}^{\prime}=-\frac{\log _{p_{\imath}}(-\mu / \lambda)}{\log _{p_{\imath}}(\alpha / \beta)}=+\frac{\log _{p_{\imath}}(-\lambda / \mu)}{\log _{p_{l}}(\alpha / \beta)}=-\theta_{\imath} \quad(i=1, \ldots, t) .
$$

Now, instead of applying the algorithm twice, we can modify it, so that it works for all $n \in \mathbb{Z}$.

Lemmas 3.2 and 4.2 remain correct if we replace $n$ by $|n|$. In Theorem 4.1, the lower bound for $n_{0}$ must be replaced by

$$
n_{0}>\max (2,|\log | \mu / \lambda|| / \log |\alpha / \beta|,|\log | \lambda / \mu|| / \log |\alpha / \beta|),
$$

and $\gamma$ has to be replaced by

$$
\gamma=\min \left(|\lambda / w|-|\mu / w||\alpha / \beta|^{-n_{0}},|\mu / w|-|\lambda / w||\alpha / \beta|^{-n_{0}}\right) .
$$

Similar modifications should be made in step (i) of Algorithm A. Further, in step (ii), $r_{l}$ should be chosen so large that

$$
\begin{gathered}
\text { if } p_{l} \neq 2 \text { then } p_{i}^{r_{t}} \geqslant N_{0} \text { and } u_{l, r_{t}} \neq 0, u_{i, r_{t}} \neq p-1 ; \\
\text { else } p_{i}^{r_{t}-1} \geqslant N_{0} \text { and } u_{i, r_{t}} \neq u_{l, r_{t}-1} ;
\end{gathered}
$$

and similar modifications have to be made in step (v). With these changes, Theorem 4.5 remains true with $n$ replaced by $|n|$.

4D. Examples. We give two examples, one of the reduction algorithm, and one example where the reduction algorithm fails, because $\theta_{l}=0$.

First Example. Let $A=6, B=1, G_{0}=1, G_{1}=4, w=1, p_{1}=2, p_{2}=11$. Then $\alpha=3+2 \sqrt{2}, \beta=3-2 \sqrt{2}, \lambda=(1+2 \sqrt{2}) / 4 \sqrt{2}, \mu=(-1+2 \sqrt{2}) / 4 \sqrt{2}$, and $\Delta=$ 32. With $n_{0}=e^{60}=1.142 \ldots \times 10^{26}$ we find from (3.1) that $C_{1}<2.49 \times 10^{20}$. With the modifications of Subsection $4 C$ we have in Theorem $4.1 \gamma>0.323$, $C_{2}<1.76, C_{3}<2.62 \times 10^{26}, C_{2} C_{3}<4.62 \times 10^{26}$. Hence, all solutions of $G_{n}=$ $2^{m_{1}} 11^{m_{2}}$ satisfy $|n|<4.62 \times 10^{26}, m_{1}, m_{2}<2.62 \times 10^{26}$. We perform the reduction algorithm step by step:

$$
\begin{aligned}
& n_{0}=2, \gamma>0.303, g_{1}=0, g_{2}=1, g>0.0275, h_{1}=-1 \\
& h_{2}=\frac{1}{2}, N_{0}=4.62 \times 10^{26}
\end{aligned}
$$


(ii)

$$
\begin{aligned}
& \theta_{1}=0.1011110111010001110010100010011000110010 \\
& 0000111101010001000001001100111010101101 \\
& 1110001011000011101000011010010101000101 \\
& 1000101011000001100101011111011010001011 \\
& 001 \ldots{ }^{*} \\
& \theta_{2}=0 . \text { A9359055307330A 1A22396230 3A006 A3366 83368 } \\
& 8270 \ldots{ }^{* *} \\
& \text { so } r_{1}=90\left(\text { since } u_{1,89}=1, u_{1,90}=0,2^{89}>N_{0}\right), r_{2}=29 \\
& \left(\text { since } u_{2,29}=6,11^{29}>N_{0}\right) .
\end{aligned}
$$

(iii) $s_{1,0}=91, s_{2,0}=30$;

(v-vii) $\quad s_{1,1}=90, s_{2,1}=29, K_{1}=$.true., $N_{1}<76.9$;

(v-vii) $s_{1,2}=10, s_{2,2}=2, K_{2}=$.true., $N_{2}<8.7$;

(v-vii) $s_{1,3}=6, s_{2,3}=1, K_{3}=$.true., $N_{3}<5.8$;

(v-vii) $s_{1,4}=6, s_{2,4}=1, K_{4}=$.false., $N_{4}<5.8$.

Hence, $|n| \leqslant 5, m_{1} \leqslant 6, m_{2} \leqslant 2$. We have

\begin{tabular}{r|rrrrrrrrrrr}
$n$ & -5 & -4 & -3 & -2 & -1 & 0 & 1 & 2 & 3 & 4 & 5 \\
\hline$G_{n}$ & 2174 & 373 & 64 & 11 & 2 & 1 & 4 & 23 & 134 & 781 & 4552
\end{tabular}

So there are 5 solutions: with $n=-3,-2,-1,0$, and 1 .

Second Example. Let $A=16, B=1, G_{0}=1, G_{1}=8, w=1, p_{1}=2, p_{2}=11$. Then $\alpha=8+3 \sqrt{7}, \beta=8-3 \sqrt{7}, \lambda=\mu=\frac{1}{2}$, so $\lambda / \mu=1$ is a root of unity, hence $\theta_{1}=\theta_{2}=0$. Notice that $\left\{G_{n}\right\}$ is of type $\left\{S_{n}\right\}$. We have

\begin{tabular}{c|rrrrrrr}
$n$ & -3 & -2 & -1 & 0 & 1 & 2 & \multicolumn{1}{c}{3} \\
\hline$G_{n}$ & 2024 & 127 & 8 & 1 & 8 & 127 & 2024 \\
$G_{n}(\bmod 16)$ & 8 & -1 & 8 & 1 & 8 & -1 & 8 \\
$G_{n}(\bmod 11)$ & 0 & 6 & 8 & 1 & 8 & 6 & 0 \\
$G_{n}\left(\bmod 11^{2}\right)$ & 88 & 6 & 8 & 1 & 8 & 6 & 88
\end{tabular}

It follows that ord ${ }_{2}\left(G_{n}\right)$ is 0 or 3 , according as $n$ is even or odd, and $\operatorname{ord}_{11}\left(G_{n}\right)>0$ if and only if $n \equiv 3(\bmod 6)$. Now, $G_{3} \mid G_{3 k}$ for all odd $k$. Notice that $11^{2}+G_{3}$, and $G_{3}$ is not 11 times a power of 2 . Hence, $G_{3}$ has an odd prime divisor different from 11 , namely 23 . It follows that $23 \mid G_{n}$ whenever $11 \mid G_{n}$. Thus $m_{2}=0$, and there remain only three solutions: for $n=-1,0$, and 1 .

We note that it is not difficult to prove that a similar argument applies whenever $\lambda / \mu= \pm 1$.

5. The Generalized Ramanujan-Nagell Equation. The most interesting application of the reduction algorithm of the preceding section seems to be the solution of the generalized Ramanujan-Nagell equation (1.2). Let $k$ be a nonzero integer, and $p_{1}, \ldots, p_{t}$ distinct prime numbers. Then we ask for all nonnegative integers $x$, $z_{1}, \ldots, z_{t}$ with

$$
x^{2}+k=p_{1}^{z_{1}} \cdots p_{t}^{z_{t}}
$$

*We write the $p$-adic number $\sum_{l=0}^{\infty} u_{l} p^{\prime}$ as $0 . u_{0} u_{1} u_{2} \ldots$

**A denotes 10. 
First we note that $z_{i}=0$ whenever $-k$ is a quadratic nonresidue $\left(\bmod p_{i}\right)$. Thus we assume that this is not the case for all $i$. Let $p_{i} \mid k$ for $i=1, \ldots, s$ and $p_{i}+k$ for $i=s+1, \ldots, t$. Let ord $p_{i}(k)$ be odd for $i=1, \ldots, r$ and even for $i=r+1, \ldots, s$. Dividing by large enough powers of $p_{i}(i=1, \ldots, s)$, (1.2) reduces to a finite number of equations

$$
D_{0} x_{1}^{2}+k_{1}=p_{r+1}^{z_{r+1}^{\prime}} \cdots p_{t}^{z_{t}^{\prime}}
$$

with $p_{1}+k_{1}(i=1, \ldots, t)$ and $D_{0}$ composed of $p_{1}, \ldots, p_{r}$ only, and squarefree. We distinguish between the $2^{t-r}$ combinations of $z_{i}^{\prime}$ odd or even $(i=r+1, \ldots, t)$. Suppose that $z_{\imath}^{\prime}$ is odd for $i=r+1, \ldots, u$ and even for $i=u+1, \ldots, t$. Put

$$
y=p_{r+1}^{\left(z_{r+1}^{\prime}-1\right) / 2} \cdots p_{u}^{\left(z_{u}^{\prime}-1\right) / 2} p_{u+1}^{z_{u+1}^{\prime} / 2} \cdots p_{t}^{z_{t}^{\prime} / 2}
$$

then, from (5.1),

$$
D_{0} x_{1}^{2}-p_{r+1} \cdots p_{u} y^{2}=-k_{1} \text {. }
$$

Put $D=D_{0} p_{r+1} \cdots p_{u}$. Then (5.2) and (5.3) lead to

$$
\left\{\begin{array}{l}
v^{2}-D w^{2}=k_{2} \\
v=p_{r+1}^{m_{r+1}} \cdots p_{t}^{m_{t}}
\end{array}\right.
$$

with $v=p_{r+1} \cdots p_{u} y, w=x_{1}, k_{2}=k_{1} p_{r+1} \cdots p_{u}$, and also to

$$
\left\{\begin{array}{l}
v^{2}-D w^{2}=k_{2}, \\
w=p_{r+1}^{m_{r+1}} \cdots p_{t}^{m_{t}},
\end{array}\right.
$$

with $v=D_{0} x_{1}, w=y, k_{2}=-k_{1} D_{0}$. We proceed with either (5.4) or (5.5), whichever is the most convenient (e.g., the one with the smaller $\left|k_{2}\right|$ ).

If $D=1$, then (5.4) and (5.5) are trivial. So assume $D>1$. Let $\varepsilon \in \mathbb{Z}+\sqrt{D} \mathbb{Z}$ be the smallest unit with $\varepsilon>1$ and $N(\varepsilon)= \pm 1$. It is well known that the solutions $v, w$ of $v^{2}-D w^{2}=k_{2}$ decompose into a finite number of classes of associated solutions. Let there be $T$ classes, and choose in the $\tau$ th class $(\tau=1, \ldots, T)$ the solution $v_{\tau, 0}$, $w_{\tau, 0}$ such that $\gamma_{\tau}=v_{\tau, 0}+w_{\tau, 0} \sqrt{D}>1$ is minimal. Then all solutions of $v^{2}-D w^{2}=$ $k_{2}$ are given by $v= \pm v_{\tau, n}, w= \pm w_{\tau, n}$, with

$$
\left\{\begin{array}{l}
v_{\tau, n}=\left(\gamma_{\tau} \varepsilon^{n}+\gamma_{\tau}^{\prime} \varepsilon^{-n}\right) / 2 \\
w_{\tau, n}=\left(\gamma_{\tau} \varepsilon^{n}-\gamma_{\tau}^{\prime} \varepsilon^{-n}\right) / 2 \sqrt{D}
\end{array}\right.
$$

for $n \in \mathbb{Z}$, where $\gamma_{\tau}^{\prime}=v_{\tau, 0}-w_{\tau, 0} \sqrt{D}$. That is, $\left\{v_{\tau, n}\right\}_{n=-\infty}^{\infty}$ and $\left\{w_{\tau, n}\right\}_{n=-\infty}^{\infty}$ are linear binary recurrence sequences. Now, (5.4) and (5.5) reduce to $T$ equations of type (1.1). If $k_{2}=1$, then $\gamma_{\tau}=\varepsilon, \gamma_{\tau}^{\prime}=\varepsilon^{-1}$. If $k_{2} \mid 2 D, k_{2} \neq 1$, then it is easy to prove that $\gamma_{\tau}^{2}=\left|k_{2}\right| \varepsilon, \gamma_{\tau}^{\prime 2}=\left|k_{2}\right| \varepsilon^{-1}$, so that

$$
\begin{aligned}
& v_{\tau, n}=\left|k_{2}\right|^{1 / 2}\left(\left(\gamma_{\tau}\left|k_{2}\right|^{-1 / 2}\right)^{2 n+1}+\left(\gamma_{\tau}^{\prime}\left|k_{2}\right|^{-1 / 2}\right)^{2 n+1}\right) / 2, \\
& w_{\tau, n}=\left|k_{2}\right|^{1 / 2}\left(\left(\gamma_{\tau}\left|k_{2}\right|^{-1 / 2}\right)^{2 n+1}-\left(\gamma_{\tau}^{\prime}\left|k_{2}\right|^{-1 / 2}\right)^{2 n+1}\right) / 2 \sqrt{D} .
\end{aligned}
$$

In both cases, (5.4) and (5.5) can be solved by elementary means (see the remarks following Lemma 4.4, and Mahler [10]). If $k_{2}+2 D$, then we apply the reduction algorithm to one of the equations $v_{\tau, n}=p_{r+1}^{m_{r+1}} \cdots p_{t}^{m_{t}}, w_{\tau, n}=p_{r+1}^{m_{r+1}} \cdots p_{t}^{m_{t}}$. Notice 
that $n$ is allowed to be negative, so we can apply the modified algorithm (see Subsection 4C, Remark 3).

Thus we have a procedure for solving (1.2). It is well known how the unit $\varepsilon$ and the minimal solutions $v_{\tau, 0}, w_{\tau, 0}(\tau=1, \ldots, T)$ can be computed by the continued fraction algorithm.

We conclude this section with an example.

THEOREM 5.1. The only nonnegative integers $x$ such that $x^{2}+7$ has no prime divisors larger than 20 are the 16 in the following table:

\begin{tabular}{ll|rl|rc|rc}
$x$ & $x^{2}+7$ & $x$ & $x^{2}+7$ & $x$ & $x^{2}+7$ & $x$ & $x^{2}+7$ \\
\hline 0 & 7 & 5 & $32=2^{5}$ & 13 & $176=2^{4} \times 11$ & 53 & $2816=2^{8} \times 11$ \\
1 & $8=2^{3}$ & 7 & $56=2^{3} \times 7$ & 21 & $448=2^{6} \times 7$ & 75 & $5632=2^{9} \times 11$ \\
2 & 11 & 9 & $88=2^{3} \times 11$ & 31 & $968=2^{3} \times 11^{2}$ & 181 & $32768=2^{15}$ \\
3 & $16=2^{4}$ & 11 & $128=2^{7}$ & 35 & $1232=2^{4} \times 7 \times 11$ & 273 & $74536=2^{3} \times 7 \times 11^{3}$
\end{tabular}

Sketch of Proof. Since -7 is a quadratic nonresidue modulo 3, 5, 13, 17, and 19, we have only the primes 2,7 , and 11 left. Only one factor 7 can occur, thus we have to solve the two equations

$$
\begin{gathered}
x^{2}+7=2^{z_{1}} 11^{z_{2}}, \\
x^{2}+7=7 \times 2^{z_{1}} 11^{z_{2}} .
\end{gathered}
$$

Equation (5.8) can be solved in an elementary way. We distinguish four cases, each leading to an equation of the type

$$
y^{2}-D z^{2}=c
$$

with $c \mid 2 D$, and either $y$ or $z$ composed of factors 2 and 11 only. We have

$$
z_{1} \text { even, } \quad z_{2} \text { even, } \quad y=2^{z_{1} / 2} 11^{z_{2} / 2}, \quad z=x / 7, \quad c=1, \quad D=7
$$

In the second example of Subsection 4D we have worked out case (i). We leave the other cases to the reader.

Equation (5.7) can be solved by the reduction algorithm. Again, we have four cases, each leading to an equation of the type

$$
y^{2}-D z^{2}=c
$$

with either $y$ or $z$ composed of factors 2 and 11 only. We have

$$
z_{1} \text { even, } z_{2} \text { even, } y=x, \quad z=2^{z_{1} / 2} 11^{z_{2} / 2}, \quad c=-7, \quad D=1
$$
$z_{1}$ odd,$\quad z_{2}$ even, $y=x, \quad z=2^{\left(z_{1}-1\right) / 2} 11^{z_{2} / 2}, \quad c=-7, \quad D=2$;

$$
z_{1} \text { even, } z_{2} \text { odd, } \quad y=x, \quad z=2^{z_{1} / 2} 11^{\left(z_{2}-1\right) / 2}, \quad c=-7, \quad D=11 \text {; }
$$

Case (i) is trivial. The other three cases each lead to one equation of type (1.1). In the first example of Subsection 4D we have worked out case (ii). With the following data the reader should be able to perform Algorithm A by hand for the cases (iii) and (iv), thus completing the proof. It will be safe to take $N<10^{30}$. 
Case (iii) $\alpha=10+3 \sqrt{11}, \lambda=(2+\sqrt{11}) / 2 \sqrt{11}$,

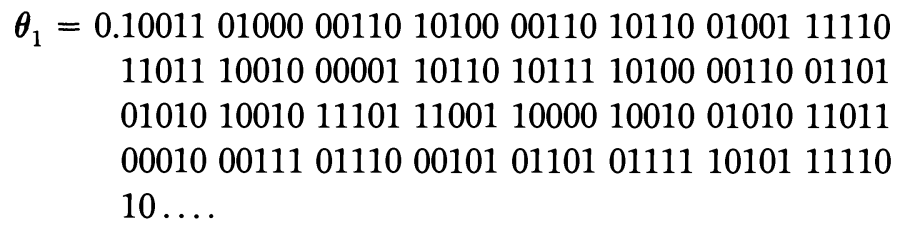

$$
\begin{gathered}
\theta_{2}=0.23075764253900426090 \text { A92A1 } 037570731458414 \\
\text { 7A238 } \ldots
\end{gathered}
$$

Case (iv) $\quad \alpha=197+42 \sqrt{22}, \lambda=(9+2 \sqrt{22}) / 2 \sqrt{22}$,

$$
\begin{array}{rl}
\theta_{1}= & 0.1110101101011100101010111100010010000011 \\
10000001101010101100011010111101101 & 10101 \\
01011 & 10100011001110110011000110001011110 \\
10101011001001111111010010111000000 & 01110 \\
011 \ldots & \\
\theta_{2}= & \text { 0.6A001 6818422921902A0 724A4 } 167694565016482 \\
\text { 5A6AA } \ldots .
\end{array}
$$

Remark. Let $\Phi(X, Y)=a X^{2}+b X Y+c Y^{2}$ be a quadratic form with integral coefficients, and $\Delta=b^{2}-4 a c$ positive or negative. Let $k$ be a nonzero integer, and $p_{1}, \ldots, p_{t}$ distinct prime numbers. Then we notice that

$$
4 a \Phi(X, Y)=(2 a X+b Y)^{2}-\Delta Y^{2},
$$

so that the diophantine equation

$$
\Phi(X, k)=p_{1}^{z_{1}} \cdots p_{t}^{z_{t}}
$$

in integers $X \neq 0, z_{1}, \ldots, z_{t} \geqslant 0$, can be solved by our method. Also the equation

$$
\Phi\left(X, p_{1}^{z_{1}} \cdots p_{t}^{z_{t}}\right)=k
$$

can be solved in this way.

Acknowledgments. We thank F. Beukers and R. Tijdeman for their comments. The first-named author was supported by a fellowship of the Alexander von Humboldt Stiftung. The second-named author was supported by the Netherlands Foundation for Mathematics (SMC) with finanical aid from the Netherlands Organization for the Advancement of Pure Research (ZWO).

KLTE Matematikai Intézet

H-4010 Debrecen Pf 12, Hungary

Mathematisch Instituut R. U. Leiden

Postbus 9512

2300 RA Leiden, The Netherlands

1. A. Baker, Transcendental Number Theory, Cambridge Univ. Press, New York, 1975.

2. F. Beukers, "On the generalized Ramanujan-Nagell equation," I: Acta Arith., v. 38, 1981, pp. 389-410; II: Acta Arith., v. 39, 1981, pp. 113-123. 
3. A. Bremner, R. Calderbank, P. Hanlon, P. Morton \& J. Wolfskill, "Two-weight ternary codes and the equation $y^{2}=4 \times 3^{\alpha}+13$," J. Number Theory, v. 16, 1983, pp. 212-234.

4. J.- H. EvertSE, "On equations in S-units and the Thue-Mahler equation," Invent. Math., v. 75, 1984, pp. 561-584.

5. H. Hasse, "Über eine diophantische Gleichung von Ramanujan-Nagell und ihre Verallgemeinerung," Nagoya Math. J., v. 27, 1966, pp. 77-102.

6. N. Koblitz, p-Adic Numbers, p-Adic Analysis and Zeta-Functions, Springer-Verlag, New York, 1977.

7. D. H. LeHMER, “On a problem of Størmer,” Illinois J. Math., v. 8, 1964, pp. 57-79.

8. F. J. MacWilliams \& N. J. A. Sloane, The Theory of Error-Correcting Codes, North-Holland, Amsterdam, 1977.

9. K. MAHLER, "Eine arithmetische Eigenschaft der rekurrierenden Reihen," Mathematika B (Leiden), v. 3, 1934, pp. 153-156.

10. K. Mahler, “Über den grössten Primteiler spezieller Polynome zweiten Grades," Arch. Math. Naturvid. B, v. 41, 1935, pp. 3-26.

11. M. MignotTE, "On the automatic resolution of certain diophantine equations," in EUROSAM 84, Proceedings, Lecture Notes in Comput. Sci., v. 174, Springer-Verlag, 1984, pp. 378-385.

12. A. Pетнӧ, "Perfect powers in second order recurrences," in Topics in Classical Number Theory, Colloq. Math. Soc. János Bolyai, vol. 34, Budapest, 1981, pp. 1217-1227.

13. А. Ретнӧ, “Full cubes in the Fibonacci sequence," Publ. Math. Debrecen, v. 30, 1983, pp. 117-127.

14. A. Ретнӧ, "On the solution of the diophantine equation $G_{n}=p^{z}$," Proceedings EUROCAL 85, Linz, Austria, Vol. 2, Lecture Notes in Comput. Sci., vol. 204, Springer-Verlag, 1985, pp. 503-512.

15. A. J. VAN DER POORTEN, "Linear forms in logarithms in the p-adic case," in Transcendence Theory: Advances and Applications (A. Baker \& D. W. Masser, eds.), Academic Press, London, 1977, pp. 29-57.

16. A. Schinzel, "On two theorems of Gelfond and some of their applications," Acta Arith., v. 13, 1967, pp. 177-236.

17. T. N. Shorey \& R. Tijdeman, Exponential Diophantine Equations, Cambridge Univ. Press, Cambridge, 1986.

18. C. Størmer, "Quelques théorèmes sur l'équation de Pell $x^{2}-D y^{2}= \pm 1$ et leurs applications," Skrifter Videnskabs-selskabet I, Math.-Naturv. Kl., 1897, pp. 1-48.

19. R. J. Stroeker \& R. Tijdeman, "Diophantine equations," in Computational Methods in Number Theory (H. W. Lenstra, Jr. \& R. Tijdeman, eds.), MC Tract 155, Amsterdam, 1982, pp. 321-369.

20. N. Tzanakis, "On the diophantine equation $y^{2}-D=2^{k}, " J$. Number Theory, v. 17, 1983, pp. 144-164.

21. N. TZanakis \& J. Wolfskill, "On the diophantine equation $y^{2}=4 q^{n}+4 q+1$," J. Number Theory. (To appear.)

22. N. TZanakis \& J. Wolfskill, "The diophantine equation $x^{2}=4 q^{a / 2}+4 q+1$ with an application in coding theory." (To appear.)

23. B. M. M. DE WeGER, "Products of prime powers in binary recurrence sequences II," Math. Comp., v. 47,1986 , pp. $729-739$. 